\title{
INVARIANT SUBSPACES ON RIEMANN SURFACES OF PARREAU-WIDOM TYPE
}

\author{
BY \\ MIKIHIRO HAYASHI
}

\begin{abstract}
In this paper we generalize Beurling's invariant subspace theorem to the Hardy classes on a Riemann surface with infinite handles. The problem is to classify all closed (weak* closed, if $p=\infty) H^{\infty}(d \chi)$-submodules, say $m$, of $L^{p}(d \chi)$, $1 \leqslant p \leqslant \infty$, where $d \chi$ is the harmonic measure on the Martin boundary of a Riemann surface $R$, and $H^{\infty}(d \chi)$ is the set of boundary functions of all bounded analytic functions on $R$. Our main result is stated roughly as follows. Let $R$ be of Parreau-Widom type, that is, the space $H^{\infty}(R, \gamma)$ of bounded analytic sections contains a nonzero element for every complex flat line bundle $\gamma \in \pi(R)^{*}$. We may assume, without loss of generality, that the Green's function of $R$ vanishes at the infinity. Set $m^{\infty}(\gamma)=\sup \left\{|f(\mathbf{O})|: f \in H^{\infty}(R, \gamma),|f| \leqslant 1\right\}$ for a fixed point $\mathbf{O}$ of $R$. Then, a necessary and sufficient condition in order that every such an $m$ takes either the form $\mathrm{m}=C_{E} L^{p}(d \chi)$, where $C_{E}$ is the characteristic function of a set $E$, or the form $m=q H^{p}(d \chi, \gamma)$, where $|q|=1$ a.e. and $\gamma$ is some element of $\pi(R)^{*}$ is that $m^{\infty}(\gamma)$ is continuous for the variable $\gamma \in \pi(R)^{*}$.
\end{abstract}

1. Introduction. This paper deals with the classification of invariant subspaces of the $L^{p}$ spaces in terms of Hardy classes on certain Riemann surfaces. Our classification theorem is closely related to direct and inverse Cauchy theorems.

In a famous paper [1], Beurling classified all closed invariant subspaces of the Hardy class $\mathrm{H}^{2}$ on the open unit disc. Though his study originated in the invariant subspace problem on Hilbert space, our classification problem is traced back to this result. There are of course several intermediate developments between the present work and Beurling's one. The case of an annulus was considered by Sarason [18]. For compact bounded Riemann surfaces, various classification theorems of invariant subspaces were given by Forelli [6], Voichick [21, 22] and Hasumi [7]. In more recent years, Neville $[\mathbf{1 1}, \mathbf{1 2}]$ succeeded in obtaining a similar classification theorem on certain plane domains with infinite connectivity, and this result was extended to certain classes of open Riemann surfaces by Hasumi [8,9] and Neville [13]. However, a problem remained because the conditions assumed on the Riemann surface are not well designed for use.

The purpose of this paper is to present the classification theorem on a more satisfactory class of Riemann surfaces, which is of course (probably, strictly) broader than those considered by Neville and Hasumi.

Received by the editors March 3, 1981 and, in revised form, November 2, 1982. 1980 Mathematics Subject Classification. Primary 30A78, 30A98; Secondary 46J15. $K e$ ' words and phrases. Invariant subspace, $H^{p}$ spaces, Riemann surfaces.

'This work was partially supported by NSF Grant Number MCS 77-02213. 
The class of Riemann surfaces we have in mind was introduced by Parreau [14]. Various considerations led Widom [23] more recently to rediscover the same class. A Riemann surface in this class will be said to be of Parreau-Widom type (cf. the next section, for a precise definition). Our classification theorem for invariant subspaces (Theorem 5.1) will be given on Riemann surfaces of Parreau-Widom type satisfying an additional condition.

In $\S 6$, we shall investigate this condition and show a way of constructing a variety of Riemann surfaces with this condition. We may emphasize this fact because it gives the first example of a Riemann surface with infinite handles on which the classification theorem of invariant subspaces is obtained; on the contrary, it is unknown, as far as we are aware, whether the class of Riemann surfaces considered by Neville and Hasumi contains a Riemann surface with infinite handles or not.

Finally, we note that the methods employed in this paper are similar to those used by Hasumi [8]. Green's lines and the Martin boundary play a fundamental role. Although it is also possible to organize the development in terms of the Hayashi boundary (homeomorphic to Wiener's harmonic boundary), which Neville used, the methods employed here give a better formulation of our classification theorem (Theorem 5.1).

ACKNOWLedgement. I would like to express my sincere thanks to Professor $M$. Hasumi for his valuable suggestions, discussions and patience during the preparation of the paper. I would like to express my sincere thanks to Professor T. W. Gamelin for his valuable discussions and constant encouragement. I am also grateful to the referees for many helpful comments, corrections and, especially, indicating to me a serious mistake which I made in relation to the condition (3.1) in a previous version of this paper.

The first draft of the present paper was written under the title "Direct and inverse Cauchy theorem and application to invariant subspaces".

2. Notations and basic facts. In this section we shall fix some notations and summarize basic known facts.

Throughout the paper $R$ will be a hyperbolic Riemann surface and $\mathbf{O} \in R$ will be a fixed point. The Green function on $R$ will be denoted by $G(z, \zeta)$. Moreover, $R^{*}$ will denote the Martin compactification of $R, \Delta$ the Martin boundary of $R$ (i.e., $\Delta^{*}=R^{*} \backslash R$ by definition), and $d \chi$ the Harmonic measure for the point $\mathbf{O}$ on $\Delta$.

Fine limit. Let $\Delta_{1}$ be the set of minimal points of $\Delta$. Then $\Delta_{1}$ has full measure with respect to $d \chi$. For each point $b \in \Delta_{1}$ a specific filter base on $R$ is defined as in [3, Abschnitt 17].

If a function $f$ on $R$ has a limit along this filter base, we will denote the limit by $\hat{f}(b)$.

The class $L P(R)$ (cf. [3, Abschnitt 2; 8, 2 ; and 13, §§2.1 and 2.2]). We shall denote by $L P(R)$ the set of differences of functions which are positive and harmonic on $R$ except for isolated logarithmic singularities with integer coefficients. Moreover, $S(R)$ (resp. $Q(R)$ ) will be the class of harmonic functions on $R$ which are singular (resp., quasi-bounded), and $B(R)$ will be the set of functions of the form

$$
u(z)=\sum_{k} n_{k} G\left(z, \zeta_{k}\right)
$$


such that $u(z)$ is finite except for $z=\zeta_{k}$, where $\left\{n_{k}\right\}$ and $\left\{\zeta_{k}\right\}$ are sets of integers and discrete points of $R$, respectively.

The following facts are known:

(2.1) Equipped with the natural addition and order relation for functions, the class $L P(R)$ becomes an ordered abelian group possessing a complete lattice structure.

(2.2) The classes $B(R), S(R)$ and $Q(R)$ are mutually orthogonal bands of $L P(R)$, and we have the orthogonal decomposition

$$
L P(R)=B(R) \oplus S(R) \oplus Q(R) .
$$

Every harmonic function of the form $u=u_{S}+u_{Q}, u_{S} \in S(R), u_{Q} \in Q(R)$, has a unique integral representation

$$
u(z)=\int_{\Delta_{1}} k_{b}(z) d \mu(b) .
$$

where $k_{b}(z)$ is the Martin kernel for $R$ and $\mu$ is a finite measure on $\Delta_{1}$ [3, Satz 13.1]. The correspondence between $u$ and $\mu$ is a vector lattice isomorphism, and $u_{S}$ (resp., $u_{Q}$ ) corresponds to the $d \chi$-singular (resp., $d \chi$-absolutely continuous) part of $\mu$ [3, Folgesatz 13.1]. Moreover,

$$
u_{Q}(z)=\int_{J_{1}} \hat{u}(b) k_{b}(z) d \chi(b)
$$

[3, Folgesatz 14.2]. This shows that $\hat{u}=0$ a.e. on $\Delta_{1}, \hat{u}=\hat{u}_{Q}$ a.e. on $\Delta_{1}$, and $\hat{u}$ is integrable with respect to $d \chi$. Moreover, if a potential $p$ is continuous on $R$, it follows from [3, Satz 14.2] that $\hat{p}=0$ a.e. on $\Delta_{1}$ with respect to $d \chi$. In summary, we have the following fact:

(2.3) Let $u=u_{B}+u_{S}+u_{Q}$, where $u_{B} \in B(R), u_{S} \in S(R)$ and $u_{Q} \in Q(R)$. Then, $\hat{u}(b)$ exists a.e. on $\Delta$. Moreover, $\hat{u}(b)=\hat{u}_{Q}(b)$ a.e. and $\hat{u}_{B}(b)=\hat{u}_{S}(b)=0$ a.e. on $\Delta$.

Multiplicative meromorphic functions of bounded characteristic. A multiple-valued meromorphic function, say $f$, is called multiplicative if $|f|$ is single valued. If, in addition, $\log |f|$ belongs to $L P(R), f$ is said to be of bounded characteristic (compare with the concept of a locally meromorphic modulus [11; 13, Chapter 2]). We shall denote by $M_{e} B(R)$ the set of multiplicative meromorphic functions of bounded characteristic, and by $M B(R)$ those which are single-valued. By definition, it is clear that $\log |f| \in L P(R)$ if $f \in M_{e} B(R)$. Conversely, we begin with $u \in L P(R)$. By (2.2), $u$ can be written in the form $u=\Sigma n_{k} G\left(\cdot, \zeta_{k}\right)+v$, where $v$ is regular. So, the additive multiple-valued harmonic conjugate of $u$, denoted by ${ }^{*} u$, is defined on $R \backslash\left\{\zeta_{k}\right\}$, and since the period around $\zeta_{k}$ of ${ }^{*} u$ is an integer multiple of $2 \pi$, the function

$$
f(z)=\exp \left(u(z)+i^{*} u(z)\right)
$$

extends to a multiplicative meromorphic function on $R$. In other words, $L P(R)$ is the set of logarithms of the moduli of the multiplicative meromorphic functions of bounded characteristic.

Inner functions (cf. [13, §2.3]). A multiplicative analytic function $f$ will be called an outer function if $\log |f| \in Q(R)$. A multiplicative analytic function $f$ is inner if $|f| \leqslant 1$ and $\log |f| \in I(R)$, where $I(R)=B(R) \oplus S(R)$. By (2.2), every function $f \in M_{e} B(R)$ 
has a unique inner-outer factorization up to multiplication by unimodular constants; say, $f=q g$, where $q$ and $g$ will be called the inner factor and the outer factor of $f$, respectively. One should note that the inner factor $q$ may be a quotient of inner functions.

Characters of multiplicative functions. We shall denote by $\pi(R)$ the fundamental group of $R$ whose base point is $\mathbf{O}$, and by $\pi(R)^{*}$ the character group of $\pi(R)$, that is, the set of all homomorphisms of $\pi(R)$ to the unit circle group. Let $f$ be a multiplicative meromorphic function on $R$; from now on throughout the paper, $f$ will be assumed to be associated with a unique branch $f_{\mathbf{O}}$ of $f$ at $\mathbf{O}$ and regarded as the analytic function obtained by analytic continuations of $f_{\mathbf{O}}$. For each closed curve $C$ starting from the point $\mathbf{O}$, the analytic continuation $f_{C}$ of $f_{\mathbf{O}}$ along $C$ is defined, and $f_{C}=\gamma_{f}(C) f_{\mathbf{o}}$, where $\gamma_{f} \in \pi(R)^{*}$ is determined uniquely by $f$. This $\gamma_{f}$ will be called the character of $f$. If $g$ is another multiplicative meromorphic function, then $f g$ is also a multiplicative meromorphic function, and $\gamma_{f g}(C)=\gamma_{f}(C) \gamma_{g}(C)$ for $C \in \pi(R)$. Moreover, if $g$ has the same character as $f$, then the sum $f+g$ is well defined and is a multiplicative meromorphic function with the same character as $f$ and $g$.

Hardy classes. Let $1 \leqslant p \leqslant \infty$ and $\gamma \in \pi(R)^{*}$. We shall denote by $H^{p}(R, \gamma)$ the set of all multiplicative analytic functions $f$ such that $f$ has the character $\gamma$, and $|f|^{p}$ has a harmonic majorant on $R(|f|$ is bounded, if $p=\infty)$. We shall also write $H^{p}(R)$ instead of $H^{p}(R, 1)$. Now we define

$$
\begin{gathered}
\|f\|_{p}=\left[\left(\text { L.H.M. } \mid f f^{p}\right)(\mathbf{O})\right]^{1 / p}, \quad 1 \leqslant p<\infty, \\
\|f\|_{\infty}=\sup _{z \in R}|f(z)|,
\end{gathered}
$$

where "L.H.M." means the "least harmonic majorant". For each $\gamma \in \pi(R)^{*}$, the class $H^{p}(R, \gamma)$ becomes a Banach space equipped with the norm $\|f\|_{p}$. It is known that $\log |f| \in L P(R)$ and it is written as

$$
\log |f|=u_{Q}-u_{I}, \quad u_{Q} \in Q(R), u_{I} \in I(R), u_{I} \geqslant 0,
$$

and that L.H.M. $|f|^{p}$ is quasi-bounded (cf. [13, Theorem 2.5.3]). In particular, the inner factor of $f$ is an inner function. Hence,

$$
|f|^{p}(z) \leqslant \exp \left(p u_{Q}(z)\right) \leqslant \int \exp \left(p \hat{u}_{Q}(b)\right) k_{b}(z) d \chi(b) .
$$

Here we have used Jensen's inequality for the integral representation $u_{Q}=$ $\int \hat{u}_{Q}(b) k_{b} d \chi(b)$. By (2.3), $|f| \hat{\mid}=\exp \hat{u}_{Q}$ a.e. on $\Delta$. This shows that

$$
\left(\text { L.H.M. }|f|^{p}\right)(z)=\int|f|^{p}(b) k_{b}(z) d \chi(b) \text {. }
$$

Therefore,

$$
\|f\|_{p}=\|\mid f \hat{\mid}\|_{p},
$$

where the right side is of course the $L^{p}$-norm with respect to the harmonic measure $d \chi$.

Parreau-Widom type. A Riemann surface $R$ is said to be of Parreau-Widom type if

$$
H^{\infty}(R, \gamma) \neq\{0\} \quad \text { for every } \gamma \in \pi(R)^{*} .
$$


The Riemann surfaces that satisfy this property were characterized by Widom [23]. In the case of a hyperbolic Riemann surface that is regular (i.e., $\{z \in R$ : $G(z, \mathbf{O}) \geqslant \varepsilon\}$ is compact for all $\varepsilon>0$ ), Parreau [14] had introduced the property

$$
\sum_{k} G\left(a_{k}, \mathbf{O}\right)<\infty
$$

where $a_{k}$ denote the zero points of the meromorphic differential $d G(\cdot, \mathbf{O})+$ $d^{*} G(\cdot, \mathbf{O})$, counting multiplicities. The points $a_{k}$ will be called the critical points of the function $G(\cdot, \mathbf{O})$. Widom showed that the two properties (2.7) and (2.8) are equivalent if $R$ is a regular hyperbolic Riemann surface ([23, p. 314]). In his paper [9] Hasumi showed that every hyperbolic Riemann surface satisfying the property (2.7) is obtained from a regular surface satisfying (2.7) merely by puncturing at a discrete set of points $w_{i}$ satisfying $\Sigma G\left(w_{i}, \mathbf{O}\right)<\infty$.

3. Direct and inverse Cauchy theorems. From now on throughout the paper, $R$ will be a regular hyperbolic Riemann surface of Parreau-Widom type unless otherwise stated. The regularity has been assumed here without loss of generality according to Hasumi's result mentioned at the end of the preceding section.

In order to state the Cauchy theorems, let us fix a few more notations. As in the preceding section, $a_{1}, a_{2}, \ldots$ denote the critical points of the function $G(\cdot, \mathbf{O})$. Define functions

$$
\lambda(z)=\sum_{k} G\left(z, a_{k}\right), \quad \Lambda(z)=\exp (-\lambda(z))
$$

and

$$
\tilde{\Lambda}(z)=\exp \left(-\lambda(z)-i^{*} \lambda(z)\right) .
$$

By (2.8), $\lambda \in B(R)$. We denote by $\tilde{\gamma}$ the character of the multiplicative analytic function $\tilde{\Lambda}(z)$. The direct Cauchy theorem now reads as follows:

TheOREM 3.1 (DIRECT CAUChy THEOREM). Let $R$ be a regular hyperbolic Riemann surface of Parreau-Widom type satisfying

$$
\exp \left(-\sum_{k} G\left(a_{k}, \mathbf{O}\right)\right)=\sup \left\{|f(\mathbf{O})|: f \in H^{1}(R, \tilde{\gamma}),\|f\|_{1} \leqslant 1\right\} .
$$

If $f$ is a meromorphic function on $R$ such that $\Lambda|f|$ has a harmonic majorant then $\hat{f}(b)$ exists a.e. on $\Delta$ and is integrable, and we have

$$
f(\mathbf{O})=\int \hat{f}(b) d \chi(b) .
$$

REMARK. We shall investigate condition (3.1) in $§ 6$.

Proof of Theorem 3.1. Let $B$ be the set of functions $k$, defined on $R$ except for the discrete set $\left\{a_{k}\right\}$, such that $|k|$ is dominated by a harmonic function on $R$ and such that $k / \Lambda$ extends to a single-valued meromorphic function on $R$. Recalling that ${ }^{*} \lambda$ is the harmonic conjugate of $\lambda$, we have

$$
B=\exp \left(i^{*} \lambda\right) H^{1}(R, \tilde{\gamma}) .
$$


Let $k \in B$. Then $k / \Lambda$ extends to a function belonging to $M B(R)$. It is easy to see that every function in $M B(R)$ can be written as a quotient of two bounded analytic functions, since $R$ is of Parreau-Widom type. Hence $(k / \Lambda)^{\wedge}(b)$ exists a.e. on $\Delta$ by (2.3). Since $\hat{\Lambda}(b)=1$ a.e. on $\Delta, \hat{k}(b)$ also exists a.e. on $\Delta$ and $\hat{k}(b)=(k / \Lambda) \hat{(}(b)$ a.e. on $\Delta$. We have $\hat{k} \in L^{1}(d \chi)$ by $(2.6)$, since $\exp \left(-i^{*} \lambda\right) k \in H^{1}(R, \tilde{\gamma})$. We norm the space $B$ by $\|k\|=\|\hat{k}\|_{1}$. Let us define a linear functional $\Phi$ on $B$ by

$$
\Phi(k)=(k / \Lambda)(\mathbf{O}) \text {. }
$$

By (3.1),

$$
\Lambda(\mathbf{O})=\sup \left\{|g(\mathbf{O})|: g \in H^{1}(R, \tilde{\gamma}),\|g\|_{1} \leqslant 1\right\} .
$$

Hence, it follows from (3.2) that $\|\Phi\|_{B} \leqslant 1$. By the Hahn-Banach extension theorem, there is a function $w \in L^{\infty}(d \chi)$ such that $\|w\|_{\infty} \leqslant 1$ and

$$
\Phi(k)=\int \hat{k}(b) w(b) d \chi(b) \quad \text { for } k \in B .
$$

In view of the facts that $\Lambda \in B$ and $\hat{\Lambda}(b)=1$ a.e. on $\Delta$, the following chain of inequalities

$$
1=\Phi(\Lambda)=\int 1 \cdot w d \chi \leqslant \int|w| d \chi \leqslant\|w\|_{\infty}=1
$$

becomes a chain of equalities. This implies $w(b)=1$ a.e. on $\Delta$. To conclude the proof, let $f$ be a meromorphic function satisfying the hypotheses. Then, $k=\Lambda f$ belongs to $B$. Since $\hat{k}(b)=\hat{f}(b)$ a.e. on $\Delta$, it follows that

$$
f(\mathbf{O})=(k / \Lambda)(\mathbf{O})=\int \hat{f} d \chi
$$

as was to be proved.

The inverse Cauchy theorem is known. We state it for the convenience of the reader.

InVerse Cauchy theorem (Hasumi [8,9]; Also CF. Neville [13]). Let $R$ be $a$ regular hyperbolic Riemann surface of Parreau-Widom type. Let $f^{*} \in L^{1}(d \chi)$. If

$$
\int f^{*}(b) \hat{h}(b) d \chi(b)=0
$$

for every meromorphic function $h$ on $R$ such that $h(\mathbf{O})=0$ and $\Lambda|h|$ is bounded, then $f^{*}$ is the boundary value of a function in $H^{1}(R)$, that is, there is $f \in h^{1}(R)$ with $\hat{f}(b)=f^{*}(b)$ a.e. on $\Delta$.

REMARK. In the case of compact bordered Riemann surfaces the above mentioned inverse Cauchy theorem is equivalent to the one proved by Read [15] and alternatively by Royden [16]; in this case, the direct Cauchy theorem becomes rather trivial. For certain infinitely connected plane domains, the direct and inverse Cauchy theorems were proved by Neville in his thesis [11]. The proofs were adapted to Riemann surfaces satisfying stronger conditions than ours by Hasumi [8] and Neville [13], in terms of the Martin boundary and the Hayashi boundary, respectively.

As remarked earlier, the inverse Cauchy theorem has been proved on the Riemann surfaces of Parreau-Widom type, and this fact was first noted by Hasumi [9]. In fact, 
Hasumi's proof of this theorem in [8] is valid for surfaces of Parreau-Widom type without any modificiation. Neville's proof of this theorem [13] is also valid if one makes some easy modifications; his proof can also be translated into terms of the Martin boundary. The two proofs, however, seem to be quite different from each other. In Hasumi's proof, Green's lines play an important role. In Neville's, it is crucial to make use of a remarkable inequality [13, Theorem 3.5.2], and Green's lines do not appear at all.

4. Radial limits and lifting. In this section, we summarize some properties of Green lines and some relations between the surface $R$ and its universal covering space, according to Parreau [14] and Hasumi $[\mathbf{8 , 9}$. We shall however reorganize the original statements.

Throughout the paper, we shall denote by $\mathcal{L}$ the set of (open) Green's lines on $R$ issuing from the point $\mathbf{O}$. The Green's star region in $R$ with respect to $\mathbf{O}$ is defined by

$$
S=\{\mathbf{O}\} \cup(\bigcup\{l: l \in \mathcal{L}\}) .
$$

Let $\left\{l_{n}\right\}$ be the set of singular Green's lines, i.e., $l$ 's in $\mathcal{L}$ such that

$$
\inf \{G(z, \mathbf{O}): z \in l\}>0 \text {. }
$$

Since we are assuming the regularity of $R$, the number of $\left\{l_{n}\right\}$ is at most countable. In fact, let $\left\{l_{m}^{*}\right\}$ be the set of Green's lines not lying in $\mathcal{L}$, all of which issue from the critical points $a_{k}$ of $G(z, \mathbf{O})$. Every Green's lines $l_{n}$ terminates at a critical point $a_{k}$ and, possibly, some of $l_{m}^{*}$ may terminate at $a_{k}$. The number of these Green's lines terminating at a critical $a_{k}$ is precisely one plus the multiplicity of the critical point $a_{k}$, and the same number of Green's lines, all of them lying in $\left\{l_{m}^{*}\right\}$, issue from the $a_{k}$. Thus, there must be at most countably many $l_{n}$ 's and $l_{m}^{*}$ 's. Incidentally, the Green's star region $S$ is a simply connected region obtained from $R$ by making cross cuts along $\left\{l_{m}^{*}\right\}$ (cf. Figure 4.1 for a plane domain with three boundary components).

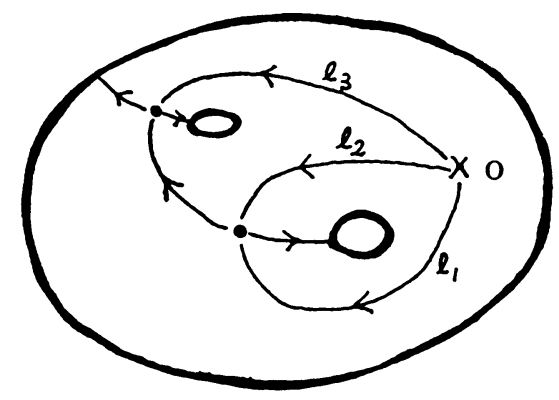

$\mathrm{R}$

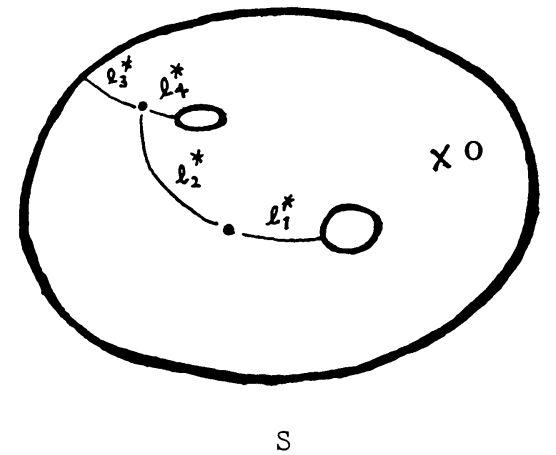

S

FigURE 4.1

If $f$ is a mapping from $l, l \neq l_{n}$, to a topological space and if $f(z)$ has a limit as $G(z, \mathbf{O}) \rightarrow 0, z \in l$, the limit will be denoted by $\check{f}(l)$ and called the radial limit of $f$ along $l$. Let $f$ be a multiplicative meromorphic function on $R$. Since $S$ is a simply connected region on $R$ containing $\mathbf{O}$, the unique analytic extension of $f_{\mathbf{O}}$ is defined 
on $S$ and will be denoted by $f_{S}$. Furthermore, we shall fix a branch of the function

$$
w(z)=\exp \left(-G(z, \mathbf{O})-i^{*} G(z, \mathbf{O})\right)
$$

on $S$, which is a one-to-one conformal mapping of $S$ into the open unit disc. Under this mapping $w(z)$, each Green line $l$ corresponds to an open radial segment $w(l)$ issuing from the origin. Assigning the argument of this segment $w(l)$ to $l$, we may identify $\varrho$ with the unit circle $\left\{e^{i \theta} ; 0 \leqslant \theta<2 \pi\right\}$. The normalized Lebesgue measure $d l=(1 / 2 \pi) d \theta$ is called the Green's measure on $\ell$.

Proposition 4.1 (ParReau [14]). Let $f$ be a multiplicative meromorphic function of bounded characteristic on $R$. Then, $\check{f}_{S}(l)$ exists a.e. on $\mathcal{L}$.

Remark. This proposition was essentially proved by Parreau [14]. He only considered the case in which $f$ is single valued, but no difficulties arise in this general case. The same proof of this proposition was rediscovered by Hasumi [9].

Proposition 4.2 (HASUmi). (a) Except for a null set $ᄅ_{0}$, every Green's line l has a single endpoint, denoted by $b_{l}$, in the Martin boundary.

(b) The mapping $\varphi: l \rightarrow b_{l}$ from $\varrho \backslash \sum_{0}$ to $\Delta$ is measurable, measure preserving, almost onto and almost one-to-one; that is, there exists a measurable subset $\mathcal{L}^{*}$ of $\mathcal{L}$

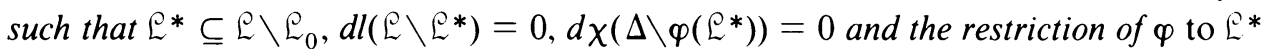
is injective.

(c) $\hat{u}\left(b_{l}\right)=\check{u}(l)$ a.e. on $\mathcal{L}^{*}$ when $u \in L P(R)$. Moreover, if $f$ is a multiplicative meromorphic function of bounded characteristic, then $|f| \hat{\mid}\left(b_{l}\right)=|\breve{f}(l)|$ a.e. on $\mathcal{L}^{*}$ and if this $f$ is also single valued, then $\hat{f}\left(b_{l}\right)=\check{f}(l)$ a.e. on $\varrho^{*}$.

Remark. The "almost one-to-one" part in (b) is not mentioned in Hasumi [8], while part (c) is mentioned only in the case when $f$ is bounded and harmonic. Thus it might be helpful to the reader to sketch briefly a proof of the proposition.

As in Hasumi [8, pp. 261-262], part (a) is proved by making use of Proposition 4.1 and l'Hospital's rule; the proof becomes simpler if we fix the point $a$ to be $\mathbf{O}$ in his proof. To prove parts (b) and (c), we first assume that $u$ is bounded harmonic. Then the two Dirichlet problems with respect to the Martin boundary with boundary data $\hat{u}(b)$ and with respect to the Green's lines with boundary data $\hat{u} \circ \varphi(l)$ have the same solution $u$ as in Brelot-Choquet [2, Théorème 30]. Making use of [3, Hilfssatz

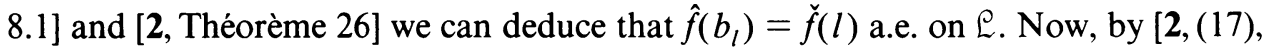
p. 247] and [3, Folgesatz 14.2], we have

$$
f(\mathbf{O})=\int_{\Delta} \hat{f}(b) d \chi(b)=\int_{\mathfrak{L}} \check{f}(l) d l .
$$

This identity implies that $\varphi$ is almost onto. The "almost one-to-one" part follows from $[14, \S 3$, Théorème]. This shows part (b). Now let $u \in L P(R), u \leqslant 0$. Then, $f=\exp \left(u+i^{*} u\right)$ is a bounded multiplicative analytic function on $R$. Put $v=$ L.H.M. $|f|$. Since $v$ is a bounded harmonic function, $\hat{v}\left(b_{l}\right)=\check{v}(l)$ a.e. on $\mathcal{L}$ as we have seen. Also, $\hat{v}(b)=|f| \hat{\mid}(b)$ a.e. on $\Delta$ by (2.5). On the other hand, by [2, Notation et Lemme 13], we have

$$
v(\mathbf{O}) \leqslant \int|\check{f}(l)| d l
$$


Since $|\check{f}(l)| \leqslant \check{v}(l)$ and since $v(\mathbf{O})=\int \check{v}(l) d l$, we see that $|\check{f}(l)|=\check{v}(l)$ a.e. on $l$. Therefore, $|\check{f}(l)|=|f| \hat{(}\left(b_{l}\right)$ and $\breve{u}(l)=\hat{u}\left(b_{l}\right)$ a.e. on $\mathcal{E}$. Now it is easy to see these identities for general $u$ and $f$ by their definitions. If $f \in M B(R)$, then condition (2.5) allows us to write $f$ as a quotient of single-valued bounded analytic functions. Hence, we have $\breve{f}(l)=\hat{f}\left(b_{l}\right)$ a.e. on $\mathcal{L}$. This completes the proof.

Throughout the paper $(U, \psi)$ will denote the universal covering space of $R$, where $U$ is the open unit disc and $\psi$ is the covering map with $\psi(0)=\mathbf{O}$. Moreover, $d \sigma$ will denote the normalized Lebesgue measure on $\partial U$ and $T=\{\tau\}$ will denote the covering transformation group for $(U, \psi)$. As is well known (cf. [3, p. 136]), the Martin compactification of $U$ is identified with the closed unit disc $U \cup \partial U$, and $\hat{\psi}\left(e^{i \theta}\right)$ exists a.e. on $\partial U$.

The following two propositions are valid for arbitrary hyperbolic Riemann surfaces and give a precise relation between the decomposition in (2.3) and the one on $U$ (cf. [13, Theorem 2.4.1]).

Proposition 4.3. Let $s$ be a positive superharmonic function, with decomposition $s=p+v+w$, where $p$ is a potential, $v \in S(R)$ and $w \in Q(R)$. Then, the decomposition of the superharmonic function $s \circ \psi$ on $U$ is given by $p \circ \psi+v \circ \psi+w \circ \psi$, that is, $p \circ \psi$ is a potential, $v \circ \psi \in S(U)$ and $w \circ \psi \in Q(U)$.

Proposition $4.4[8, \S 6]$. (a) There is a T-invariant Borel subset of of $\partial U$ such that $\sigma\left({ }^{\circ}\right)=1$ and $\hat{\psi}$ maps $(1)$ into $\Delta$.

(b) If $v \in Q(R)$, then $v \circ \psi \in Q(U)$ and

$$
(v \circ \psi) \hat{n}\left(e^{i \theta}\right)=\hat{v} \circ \hat{\psi}\left(e^{i \theta}\right) \text { a.e. on } \partial U \text {. }
$$

By this correspondence $\hat{v} \rightarrow \hat{v} \circ \hat{\psi}$, the space $L^{p}(d \chi)$ is isometrically isomorphic to $L^{p}(d \sigma)_{T}$ for $1 \leqslant p \leqslant \infty$, where $L^{p}(d \sigma)_{T}$ denotes the subspace of functions in $L^{p}(d \sigma)$ which are $T$-invariant on $\partial U$.

REMARK. An inaccurate statement exists in the proof of this fact [8, Lemma 6.2] but can be amended without difficulty.

As in $[13, \S 2.4]$, for each multiplicative meromorphic function $f$ on $R$, there exists a meromorphic function $F$ on $U$ such that $|f| \circ \psi=|F|$. We can also take this $F$ so that $f_{\mathbf{O}} \circ \psi$ agrees with the germ of $F$ at the origin. Under this convention, $F$ is uniquely determined by $f$ and we shall write $F=f \circ \psi$. Conversely, if $F$ is a meromorphic function on $U$ such that $|F|$ is $T$-invariant, then there exists a unique multiplicative meromorphic function $f$ on $U$ such that $f \circ \psi=F$.

Let $\gamma_{f}$ be the character of $f$. For each $\tau \in T$, let $C_{\tau}$ be the image of a continuous curve from 0 to $\tau(0)$ by $\psi$. Then, $\tau \rightarrow C_{\tau}$ induces a natural isomorphism of the group $T$ onto the group $\pi(R)$, and we see that

$$
F \circ \tau=\gamma_{f}\left(C_{\tau}\right) F \text { for all } \tau \in T .
$$

COROLLARY 4.5. If $f$ is a multiplicative meromorphic function of bounded characteristic on $R$, then

$$
\left|\hat{F}\left(e^{i \theta}\right)\right|=\left|f \hat{\mid} \circ \hat{\psi}\left(e^{i \theta}\right)=\right| f\left(\varphi^{-1} \circ \hat{\psi}\left(e^{i \theta}\right)\right) \mid \text { a.e. on } \partial U,
$$

where $F=f \circ \psi$. 
Proof. Note that part (b) of the proposition implies the image of $\hat{\psi}$ has full measure in $\Delta$. Thus by Propositions 4.4(a) and 4.2(b), $\varphi^{-1} \circ \hat{\psi}\left(e^{i \theta}\right)$ is defined a.e. on $\partial U$. Now let $f=q g$ be an inner-outer factorization, where $\log |q| \in I(R)$ and $\log |g| \in Q(R)$. Then, $|F|=(|q| \circ \psi)(|g| \circ \psi)$ and by Proposition 4.3, $\log |q| \circ$ $\psi \in I(U)$ and $\log |g| \circ \psi \in Q(U)$. By (2.3) and part (b) of the proposition, $\left|\hat{F}\left(e^{i \theta}\right)\right|=|g| \hat{} \circ \hat{\psi}\left(e^{i \theta}\right)=|f| \hat{} \circ \hat{\psi}\left(e^{i \theta}\right)$ a.e. on $\partial U$. Hence, the first equality holds. The second equality follows from Proposition 4.2(c).

5. Classification of invariant subspaces. By Proposition 4.2(b), we have an almost bijective mapping $l \rightarrow b_{l}$ between the Green's lines on $R$ and the points on the Martin boundary $\Delta$. For a function $f \in M_{e} B(R)$, we define a measurable function $\hat{f}(b)$ on $\Delta$ by $\hat{f}(b)=\check{f}_{S}(l)$, when $\check{f}_{S}(l)$ exists for $b=b_{l}$, and $\hat{f}(b)=0$ otherwise. When $f \in M B(R)$, the notation $\hat{f}(b)$ has been defined in a different way in $\S 2$. Two definitions of $\hat{f}(b)$, however, agree on $\Delta$ except for a null set by Proposition 4.2(c), and we may interpret $\hat{f}(b)$ in either sense if $f \in M B(R)$.

Now, by Proposition 4.1, we put

$$
H^{p}(d \chi, \gamma)=\left\{\hat{f}: f \in H^{p}(R, \gamma)\right\} .
$$

We write $H^{p}\left(d_{\chi}\right)$ instead of $H^{p}(d \chi, 1)$. The identity (2.6) and Proposition 4.2 imply that $H^{p}(d \chi, \gamma)$ is a closed subspace of $L^{p}(d \chi)$ for $1 \leqslant p \leqslant \infty$, and since $H^{\infty}(d \chi, \gamma)$ $=L^{\infty}(d \chi) \cap H^{1}(d \chi, \gamma), H^{\infty}(d \chi, \gamma)$ is weak* closed in $L^{\infty}(d \chi)$ by [5, Chapter IV, Lemma 2.1]. A linear subspace $m$ of $L^{p}(d \chi)$ is called an invariant subspace if $f \mathfrak{m} \subseteq \mathfrak{m}$ for every $f \in H^{\infty}(R)$, that is, if $\mathfrak{m}$ is is an $H^{\infty}(d \chi)$-submodule of $L^{p}(d \chi)$. Two typical examples of closed invariant subspaces are $q H^{p}(d \chi, \gamma)$ and $C_{E} L^{p}(d \chi)$, where $q \in L^{\infty}(d \chi)$ satisfies $|q|=1$ a.e. on $\Delta$, and where $C_{E}$ denotes the characteristic function of a measurable subset $E$ of $\Delta$. What we are about to prove is that every closed (weak* closed, if $p=\infty$ ) invariant subspace has one of these two forms.

In order to state our theorem more exactly, let us call attention to common inner divisors of $H^{p}(R, \gamma)$. Let $f \in M_{e} B(R)$. A quotient $q$ of inner functions is said to be an inner divisor of $f$ if the inner factor of $f / q$ is bounded. This occurs precisely when the component of $\log |f|$ in $I(R)=B(R) \oplus S(R)$ is dominated by $\log |q|$. When $\widehat{y}$ is a subset of $M_{e} B(R), q$ is said to be a common inner divisor of $\mathscr{F}$ if $q$ is a divisor of each function $f \in \mathscr{F}$. Now the class $H^{p}(R, \gamma)$ will be called regular if no nonconstant inner function is a common inner divisor of $H^{p}(R, \gamma)$. It remains as an interesting problem whether the class $H^{\infty}(R, \gamma)$ is regular for every $\gamma \in \pi(R)^{*}$ when $R$ is a regular hyperbolic Riemann surface of Parreau-Widom type. In this problem, "regularity of $R$ " is necessary, for there exists a nonregular $H^{\infty}(R, \gamma)$ whenever the surface $R$ of Parreau-Widom type is not regular. (For instance, take as $\gamma$ the character of $\exp \left(-\left(G(z, w)+i^{*} G(z, w)\right) / 2\right)$ for a nonregular point $w$ for $R$; existence of such $w$ is proved in [9].)

Finally, we put

$$
H_{0}^{p}(d \chi)=\left\{\hat{f}: f \in H^{p}(R), f(\mathbf{O})=0\right\} .
$$

An invariant subspace $m$ is called doubly invariant if $H_{0}^{\infty}(d \chi) m$ is dense (weak* dense, if $p=\infty$ ) in $m$. An invariant subspace $m$ is simply invariant if $m$ is not doubly invariant. 
Now our classification theorem reads as follows.

THEOREM 5.1. Suppose that $R$ is a regular hyperbolic Riemann surface of ParreauWidom type satisfying the identity (3.1). Let $1 \leqslant p \leqslant \infty$, and let $m$ be a closed (weak* closed, if $p=\infty)$ invariant subspace of $L^{p}(d \chi)$. Then, $m$ has one of the following forms:

(a) $m=C_{E} L^{p}(d \chi)$, where $C_{E}$ is the characteristic function of a measurable subset $E$ of the Martin boundary $\Delta$. The set $E$ is uniquely determined up to a null set by $m$.

(b) $m=q H^{p}(d \chi, \gamma)$, where $q$ is a measurable function on $\Delta$ such that $|q|=1$ a.e., and $\gamma \in \pi(R)^{*}$. The character $\gamma$ can be chosen so that $H^{p}(R, \gamma)$ is regular, and if this is the case, such a character $\gamma$ and the function $q$ are uniquely determined (up to a null set and multiplication by a unimodular constant) by $\mathrm{m}$.

Moreover, $\mathfrak{m}$ is in the form (a) (resp., (b)) if and only if $\mathfrak{m}$ is doubly (resp., simply) invariant.

REMARK. We shall investigate condition (3.1) in $\$ 6$.

The proof of the theorem is preceded by three lemmas. The first two lemmas are valid for arbitrary hyperbolic Riemann surfaces.

LeMmA 5.2. If $h \in M_{e} B(R)$ and $h \neq 0$, then $\hat{h}(b) \neq 0$ a.e. on $\Delta$.

Proof. Since $\log |h| \in L P(R)$, we have $\log |h| \hat{\in} \in L^{1}(d \chi)$ by (2.3) and the Poisson integral representation preceding it. Hence, $\hat{h}(b) \neq 0$ a.e. on $\Delta$ by Proposition $4.2(\mathrm{c})$. 4.2(c).

LEMma 5.3. Let $f \in M_{e} B(R)$. If the inner factor of $f$ is bounded on $R$, and if $|f| \hat{\mid}(b)$ is integrable, then $|f|$ has a harmonic majorant on $R$.

This lemma can be proved in a way similar to the case of the open unit disc. It depends on the inequality between arithmetic and geometric means (cf. [4, Theorem 2.8]).

If $m$ is a subset of $L^{p}(d \chi), m^{\perp}$ will denote the orthogonal complement $\{g \in$ $L^{p^{\prime}}(d \chi): \int f g d \chi=0$ for all $\left.f \in \mathbb{m}\right\}$, where $p^{\prime}$ is the conjugate exponent of $p$, i.e., $1 / p+1 / p^{\prime}=1$.

LEMma 5.4. Let $K^{1}(R)$ be the set of meromorphic functions such that $f(\mathbf{O})=0$ and $\Lambda|f|$ has a harmonic majorant. Set $K^{1}(d \chi)=\left\{\hat{f}: f \in K^{1}(R)\right\}$. Then,

$$
K^{1}(R)=\Lambda_{*} H^{1}\left(R, \gamma_{*}^{-1}\right),
$$

where $\Lambda_{*}(z)=\exp \left(-G(z, \mathbf{O})-i^{*} G(z, \mathbf{O})\right) / \tilde{\Lambda}(z)$ and $\gamma_{*}$ is the character of $\Lambda_{*}$. Moreover, if $R$ satisfies (3.1), then

$$
K^{1}(d \chi)=H^{\infty}(d \chi)^{\perp} .
$$

Proof. The first identity is easy to see by definition. Thanks to the direct and inverse Cauchy theorems in $\S 3$, the orthogonal complement in $L^{\infty}(d \chi)$ of $K^{1}(d \chi)$ is equal to $H^{\infty}(d \chi)$. Since $\Lambda_{*}$ is a quotient of inner functions, $K^{1}(d \chi)$ is a closed subspace of $L^{1}(d \chi)$. Hence, $K^{1}(d \chi)$ is the orthogonal complement of $H^{\infty}(d \chi)$.

Now we are in position to prove the theorem. 
Proof of Theorem 5.1. The notations defined in the preceding section will be used without notice. Let $\{m\}=\left\{f^{*} \circ \hat{\psi}: f^{*} \in \mathfrak{m}\right\}$, and let $\{m\}_{p}$ be the closure (weak* closure, if $p=\infty)$ in $L^{p}(d \sigma)$ of the set $\left\{F G: F \in H^{\infty}(d \sigma)\right.$ and $\left.G \in\{m\}\right\}$. Clearly $\{m\}_{p}$ is an invariant subspace of $L^{p}(d \sigma)$ with respect to $H^{\infty}(d \sigma)$. Further, $\{\mathfrak{m}\}_{p}$ is $T$-invariant, i.e, $G \circ \tau \in\{\mathfrak{m}\}_{p}$ whenever $G \in\{\mathfrak{m}\}_{p}$ and $\tau \in T$. Now, we divide the proof into three parts.

Part one. Suppose that $\{m\}_{p}$ is a doubly invariant subspace of $L^{p}(d \sigma)$. We shall prove that $m$ is in the form (a).

By [19], there is a measurable subset $L$ of $\partial U$ such that $\{m\}_{p}=C_{I} L^{p}(d \sigma)$. Since $\{m\}_{p}$ is $T$-invariant and $T$ is countably generated, $L$ may be chosen so that $L$ is $T$-invariant. By Proposition 4.4(b), there is a measurable subset $E$ of $\Delta$ such that $C_{E} \circ \hat{\psi}=C_{L}$ a.e. on $\partial U$. Clearly, $C_{E} L^{p}(d \chi)$ includes $m$. In order to show the reverse inclusion, let $p^{\prime}$ be the conjugate exponent of $p$. Suppose that $s^{*} \in L^{p^{\prime}}(d \chi)$ and $s^{*} \perp \mathrm{m}$. Take an element $f^{*}$ in $\mathrm{m}$. Since $s^{*} f^{*}$ is orthogonal to $H^{\infty}(d \chi), s^{*} f^{*} \in$ $K^{1}(d \chi)$ by Lemma 5.4. Let us choose a nonzero bounded analytic function $h \in$ $\Lambda H^{\infty}\left(R, \tilde{\gamma}^{-1}\right), h \neq 0$. Then, $\hat{h s}^{*} f^{*} \in H_{0}^{1}(d \chi)$. So, for any $F \in H^{\infty}(d \sigma)$, we have

$$
\int_{\partial U}\left(\left[\hat{h}^{*}\right] \circ \hat{\psi}\right)\left(f^{*} \circ \hat{\psi}\right) F d \sigma=\int_{\partial U}\left(\left[\hat{h} s^{*} f^{*}\right] \circ \hat{\psi}\right) F d \sigma=0 .
$$

Since the function $f^{*} \in \mathrm{m}$ is arbitrary, $\left[\hat{h} s^{*}\right] \circ \hat{\psi}$ is orthogonal to $\{\mathrm{m}\}_{p}$. Thus, $\left[\hat{h s^{*}}\right] \circ \hat{\psi}$ must vanish on the set $L$ a.e., or $\hat{h} s^{*}$ vanishes a.e. on $E$. Since $\hat{h}(b) \neq 0$ a.e. on $\Delta$ by Lemma $5.2, s^{*}=0$ on $E$ a.e. Therefore, $s^{*}$ is orthogonal to $C_{E} L^{p}(d \chi)$, and we obtain $\mathrm{m}=C_{E} L^{p}(d \chi)$. It is clear that the set $E$ is uniquely determined by $m$ up to a null set.

Part two. Suppose that $\{m\}_{p}$ is simply invariant. We shall prove that $m$ has the form (b). The proof of this part is divided into three steps.

(Step 1) We shall find the function $q$. By [20], there exists a function $Q \in L^{\infty}(d \sigma)$ such that $|Q|=1$ a.e. on $\partial U$ and $\{m\}_{p}=Q H^{p}(d \sigma)$. Let $\tau \in T$. Since $\{m\}_{p}$ is $T$-invariant, it follows from the uniqueness of $Q$ that $Q \circ \tau=\gamma(\tau)^{-1} Q$ a.e. on $\partial U$ for some unimodular complex number $\gamma(\tau)$. Clearly, $\gamma\left(\tau_{1} \tau_{2}\right)=\gamma\left(\tau_{1}\right) \gamma\left(\tau_{2}\right)$ for $\tau_{1}, \tau_{2} \in T$. Since the covering transformation group $T$ is naturally isomorphic to $\pi(R)$, we may regard $\gamma$ as a character of $\pi(R)$. Let $f^{*}$ be an element of $m$. Then, $f^{*} \circ \hat{\psi}=Q \hat{F}$ for some $F \in H^{p}(U)$. Since $f^{*} \circ \hat{\psi}$ is $T$-invariant, it follows that $F \circ \tau=\gamma(\tau) F$ for every $\tau \in T$. Therefore, there is a unique multiplicative analytic function $f$ with $f \circ \psi=I$. Here we recall our convention that $f_{\mathbf{0}} \circ \psi$ agrees with the germ of $G$ at the origin. By (4.1), the character of $f$ is $\gamma$. Since L.H.M. $\mid F^{p}$ is $T$-invariant, $|f|^{p}$ has a harmonic majorant. Hence, $f \in H^{p}(R, \gamma)$. Now suppose, in addition, that $f^{*}$ is not a null function. Set

$$
q(b)=f^{*}(b) / \hat{f}(b) \quad \text { a.e. on } \Delta .
$$

If we knew $\hat{f} \circ \hat{\psi}=\hat{F}$, it would be clear that $q \circ \hat{\psi}=Q$, so $q$ would be independent of $f^{*}$. Unfortunately, this is not true in general $(\hat{f} \circ \psi$ is $T$-invariant but $\hat{F}$ is not unless $\gamma=1)$. So fix a nonzero function $g$ of $H^{\infty}\left(R, \gamma^{-1}\right)$ and put $G=g \circ \psi$. Since $(f g)_{S}=f_{S} g_{S}$ on the Green's star region $S$, we see that $\hat{f} \hat{g}=(f g)^{\wedge}$ a.e. on $\Delta$. Since $f g^{\bullet} \in H^{p}(R),(f g)^{\wedge} \circ \hat{\psi}=\left((f g)^{\wedge} \circ \psi\right)^{\wedge}$ a.e. on $\partial U$ by Proposition $4.4(\mathrm{~b})$. Furthermore, the germ of $F G$ at the origin agrees with $(f g)_{0} \circ \psi$, so $F G=(f g) \circ \psi$, and 
hence $(F G)^{\hat{n}}=(f g)^{\hat{0}} \circ \hat{\psi}$. Thus $(\hat{f} \circ \hat{\psi})(\hat{g} \circ \hat{\psi})=(\hat{f} \hat{g}) \circ \hat{\psi}=(f g)^{\hat{\gamma}} \circ \hat{\psi}=(F G)^{\hat{\psi}}=$ $\hat{F} \hat{G}$ a.e. on $\partial U$. Hence, $f^{*} \circ \hat{\psi} / \hat{f} \circ \hat{\psi}=Q \hat{F}(\hat{g} \circ \hat{\psi}) /(\hat{f} \circ \hat{\psi})(\hat{g} \circ \hat{\psi})=Q \hat{F}(\hat{g} \circ \hat{\psi}) / \hat{F} \hat{G}$ $=Q(\hat{g} \circ \hat{\psi}) / \hat{G}$. Thus,

$$
q \circ \hat{\psi}=Q(\hat{g} \circ \hat{\psi}) / \hat{G} \quad \text { a.e. on } \partial U \text {. }
$$

This identity shows that $q$ is independent of the choice of nonzero $f^{*} \in m$. Corollary 4.5 implies that $|q|=1$ a.e. on $\Delta$. Now, by (5.1), each element $f^{*} \in m$ is written in the form

$$
f^{*}(b)=q(b) \hat{f}(b) \quad \text { a.e. on } \Delta, \text { where } f \in H^{p}(R, \gamma) \text {. }
$$

In particular, $\mathrm{m} \subseteq q H^{p}(d \chi, \gamma)$.

(Step 2) We shall prove that the last inclusion is indeed an identity. To do this, we first show that if an inner function, say $q^{\prime}$, is a common inner divisor of the set

$$
X=\left\{f \in H^{p}(R, \gamma): q \hat{f} \in \mathrm{m}\right\} .
$$

then $q^{\prime}$ is a unimodular constant. In fact, if $f^{*} \in \mathfrak{m}$, we have by (5.3)

$$
f^{*} \circ \hat{\psi}=Q \hat{F}
$$

where $f \in H^{p}(R, \gamma)$ and $F=f \circ \psi$. By Proposition 4.3, $Q^{\prime}=q^{\prime} \circ \psi$ is an inner divisor of $F$. Since this is true for every such $F$, we must have

$$
\{\mathrm{m}\}_{p} \subseteq Q \hat{Q}^{\prime} H^{p}(d \sigma),
$$

and the reverse inclusion trivially holds. By the uniqueness of function $Q, \hat{Q}^{\prime}$ must be a unimodular constant. Hence $Q^{\prime}$ and thus $q^{\prime}$ itself must be a unimodular constant. In particular, we have also shown that $H^{p}(R, \gamma)$ is regular.

Now let $s^{*}$ be an element of $L^{p^{\prime}}(d \chi)$ which is orthogonal to $m$ and let $g$ be an arbitrary element of $H^{p}(R, \gamma)$. Choosing a nonzero element $f^{*}$ of $\mathrm{m}$, put $f^{*}=q \hat{f}$. Since $m$ is invariant, $s^{*} f^{*}$ is orthogonal to $H^{\infty}(d \chi)$. Thus $s^{*} f^{*} \in K^{1}(d \chi)$. Since $f$ and $g$ have the same character, the right-hand side of the identity

$$
s^{*} q \hat{g}=s^{*} f^{*}(\hat{g} / \hat{f})
$$

is the boundary values of a meromorphic function, say $h$, of bounded characteristic. Since the left-hand side of (5.4) is independent of the choice of $f^{*} \in \mathrm{m}$, and since Lemma 5.2 guarantees that a meromorphic function of bounded characteristic is determined by its boundary values, $h$ is independent of the choice of $f^{*}$.

Let us write the inner factor of $h$ as $\Lambda_{*}\left(q_{1} / q_{2}\right)$, where $q_{1}, q_{2}$ are inner functions. The function $\Lambda_{*}$ appears because $s^{*} f^{*} \in K^{1}(d \chi)$, and the denominator $q_{2}$ comes from the function $f$ in (5.4). Hence, $q_{2}$ must be an inner divisor of $f$. As we have seen, $q_{2}$ must be a unimodular constant. Now, it follows from Lemma 5.3 that $\Lambda|h|$ has a harmonic majorant. By Lemma 5.4, we conclude that $h \in K^{1}(R)$. Since

$$
\int s^{*} q \hat{g} d \chi=\int \hat{h} \cdot 1 d \chi=0 .
$$

$s^{*}$ is orthogonal to $q H^{p}(d \chi, \gamma)$, as was to be proved.

(Step 3) We now only have to prove uniqueness of function $q$ and character $\gamma$. Let $\mathrm{m}=q^{\prime} H^{p}\left(d \chi, \gamma^{\prime}\right)$ be another expression, where $H^{p}\left(d \chi, \gamma^{\prime}\right)$ is regular. Let $f$ be a nonzero element of $H^{p}(R, \gamma)$. Then, there is an element $f^{\prime}$ of $H^{p}\left(R, \gamma^{\prime}\right)$ with 
$q \hat{f}=q^{\prime} \hat{f}^{\prime}$, or $q / q^{\prime}=\hat{f}^{\prime} / \hat{f}$. Since $\left|q / q^{\prime}\right|=1$ a.e. on $\Delta, f^{\prime} / f$ is a quotient of inner functions. By Lemma 5.2, the identity $\left(\hat{f}^{\prime} / \hat{f}\right) H^{p}\left(d \chi, \gamma^{\prime}\right)=H^{p}(d \chi, \gamma)$ implies the identity

$$
\left(f^{\prime} / f\right) H^{p}\left(R, \gamma^{\prime}\right)=H^{p}(R, \gamma)
$$

Since the members of the right-hand side are multiplicative analytic functions, the inner factor of $f^{\prime} / f$ must be bounded by the regularity of $H^{p}\left(R, \gamma^{\prime}\right)$. Similarly, the inner factor of $f / f^{\prime}$ must be bounded. Therefore, the function $f^{\prime} / f$ is a constant of modulus one, and hence, $\gamma=\gamma^{\prime}$.

Part three. Now we shall show the final part of the theorem. Suppose that $\mathrm{m}=q H^{p}(d \chi, \gamma)$, where $|q|=1$ a.e. Choose a function $g \in H^{\infty}\left(d \chi, \gamma^{-1}\right)$ with $|g|(\mathbf{O}) \neq 0$. By the direct Cauchy theorem (Theorem 3.1), we see that the function $\hat{g} / q \in L^{\infty}(d \chi)$ is orthogonal to $H_{0}^{\infty}(d \chi) \mathfrak{m}$ but not to $\mathrm{m}$. Hence, $\mathfrak{m}$ is simply invariant.

Next, we suppose that $m=C_{E} L^{p}(d \chi)$. Choose a nonzero function $f^{*}$ of $H_{0}^{\infty}(d \chi)$. Set $E_{n}=\left\{b \in \Delta:\left|f^{*}(b)\right| \geqslant 1 / n\right\}$. We define functions $g_{n}^{*}$ on $\Delta$ by $g_{n}^{*}(b)=1 / f_{n}^{*}(b)$ if $b \in E_{n}$, otherwise $g_{n}^{*}(b)=0$. Since $m$ is an $L^{\infty}(d \chi)$-module, we have

$$
f^{*} \mathfrak{m} \supseteq f^{*} g_{n}^{*} C_{E_{n}} \mathfrak{m}=C_{\left(E_{n} \cap E\right)} L^{p}(d \chi) .
$$

Letting $n \rightarrow \infty$, it follows that $f^{*} \mathrm{~m}$ is dense (weak* dense, if $p=\infty$ ) in $\mathrm{m}$. Hence, $m$ is doubly invariant. This completes the proof.

REMARK. The fact that $C_{E} L^{p}(d \chi)$ is doubly invariant was indicated to me together with its proof by M. Hasumi. Also, he has known the fact proved in Part one independently; the direct Cauchy theorem is not necessary in the proof of this part.

In the above proof, the results that have been proved without using condition (3.1) are valid even for arbitrary of Riemann surfaces of Parreau-Widom type. We have used it twice in the proof. First it has been used, as the form of Lemma 5.4, at the end of (Step 2) of Part two and second, as the form of the direct Cauchy theorem, at Part three to show that $q H^{p}(d \chi, \gamma)$ is simply invariant. However, the latter fact can be seen without using the direct Cauchy theorem. Namely, we have the following:

TheOREM 5.1'. Let $R$ be a regular hyperbolic Riemann surface of Parreau-Widom type, and let $\mathfrak{m}$ be a closed (weak ${ }^{*}$ closed if $p=\infty$ ) invariant subspace of $L^{p}(d \chi)$, $1 \leqslant p \leqslant \infty$. Then, either (a) or (b) holds:

(a) $\mathfrak{m}=C_{E} L^{p}(d \chi)$, where $E$ is a measurable subset of $\Delta$;

(b) $\mathfrak{m} \subseteq q H^{p}(d \chi, \gamma)$, where $q \in L^{\infty}(d \chi)$ and $|q|=1$ a.e.

When the case (b) occurs, the set $X=\left\{f \in H^{p}(R, \gamma): q \hat{f} \in \mathfrak{m}\right\}$ can be chosen to be regular, that is, $X$ admits no nonconstant inner function as its common inner divisor, and in this case the character $\gamma$ and the function $q$ are uniquely determined (up to a null set and multiplication by a unimodular constant) by m. Moreover, the case (a) (resp., (b)) occurs if and only if $m$ is doubly (resp., simply) invariant.

Proof. Replacing $H^{p}(R, \gamma)$ by $X$, the uniqueness of $\gamma$ and $q$ can be seen similarly. Thus, it suffices to show that $m$ is simply invariant in the case (b). Suppose that $\mathfrak{m} \subseteq q H^{p}(d \chi, \gamma)$ and the set $X$ is regular. Let $\mathfrak{m}^{\prime}$ be the (weak*) closure of 
$H_{0}^{\infty}(d \chi) m$. Then, $\mathfrak{m}^{\prime} \subseteq \hat{Z} q H^{p}\left(d \chi, \gamma \gamma_{\mathbf{o}}^{-1}\right)$, where $Z(z)=\exp \left(-G(z, \mathbf{O})-i^{*} G(z, \mathbf{O})\right)$ and $\gamma_{0}$ is the character of $Z$. Since $X$ is regular, the inner function $Z$ is not a common inner divisor of $X$. So there is an element $f \in X$ with $|f(\mathbf{O})| \neq 0$. If $q \hat{f} \in \mathrm{m}^{\prime}$, then $q \hat{f}=\hat{Z} q \hat{g}$ for some $g \in H^{p}\left(R, \gamma \gamma_{\mathbf{o}}^{-1}\right)$, but this is impossible because $f=Z g$ vanishes at $\mathbf{O}$. Hence, $q \hat{f} \notin \mathrm{m}^{\prime}$ and $q \hat{f} \in \mathfrak{m}$, as was to be proved.

The following fact has also been shown in the proof of Theorem 5.1.

COROLlaRY 5.5. If $m$ is an invariant subspace of the form $m=q H^{p}(d \chi, \gamma)$, where $|q|=1$ a.e. and $H^{p}(R, \gamma)$ is regular, then the orthogonal complement $\mathrm{m}^{\perp}$ of $\mathrm{m}$ is given by

$$
\mathrm{m}^{\perp}=\bar{q} \Lambda_{*} H^{p^{\prime}}\left(d \chi, \gamma_{*}^{-1} \gamma^{-1}\right)
$$

where $p^{\prime}$ is the conjugate exponent of $p$, i.e, $p^{-1}+p^{-1}=1$.

COROllaRy 5.6. Let $M$ be a family consisting of functions in $M_{e} B(R)$ which have the same character $\gamma$. Suppose $\{\hat{f}: f \in M\} \subseteq L^{p}(d \chi)$ for some $1 \leqslant p \leqslant \infty$. Let $[M]_{p}$ denote the closed (weak* closed, if $p=\infty$ ) invariant subspace in $L^{p}(d \chi)$ generated by $\{\hat{f}: f \in M\}$.

(a) If $M$ has no common inner divisors, more precisely, if the set of $I(R)$-parts of $\log |f|, f \in M$, is not bounded above in the lattice $L P(R)$, then $[M]_{p}=L^{p}(d \chi)$.

(b) If $M$ has a common inner divisor, then $[M]_{p}=q H^{p}\left(d \chi, \gamma \xi^{-1}\right)$, where $q$ is the greatest common inner divisor of $M$ and $\xi$ is the character of $q$. Moreover the class $H^{p}\left(R, \gamma \xi^{-1}\right)$ is regular.

REMARK. A quotient $q_{1}$ of inner functions is said to be greater than or equal to another $q_{2}$, if $q_{2}$ is a divisor of $q_{1}$, i.e., $\log \left|q_{2}\right| \geqslant \log \left|q_{1}\right|$. The greatest common inner divisor has been defined in the corollary according to this order relation. In assertion (b), $q=\vee(\log |f|)_{B}+\vee(\log |f|)_{S}$, where the suprema are taken in the lattice $L P(R)$ over the set of all $f \in M$.

Proof of Corollary 5.6. The assertion (b) follows from Part two of the proof of Theorem 5.1. In order to see the assertion (a), we suppose that $[M]_{p}$ was simply invariant. Then, $[M]_{p}=q H^{p}(d \chi, \gamma)$, where $|q|=1$ a.e. and $\gamma \in \pi(R)^{*}$. Let $g \in M$, $g \neq 0$. Then, $\hat{g}=q \hat{f}$ for some $f \in H^{p}(R, \gamma)$. Hence, $q=\hat{g} / \hat{f}$ is the boundary values of multiplicative meromorphic function $g / f$ of bounded characteristic. Let $h=g / f$. Then, $h$ is independent of $f$ and $g$ by Lemma 5.2, and we have $g=h f$. Hence $M$ must have a common inner factor, a contradiction. Thus, we must have $[M]_{p}=C_{E} L^{p}(d \chi)$ for some measurable subset $E$ of $\Delta_{1}$. By Lemma 5.2, $g$ does not vanish on a set of positive measure if $g \in M$ and $g \neq 0$. Hence, we obtain $[M]_{p}=L^{p}(d \chi)$.

Our classification theorem is stated in terms of the Martin boundary. As for the invariant subspaces of $H^{p}(d \chi)$, we can state the result without the notion of ideal boundaries as done by Neville $[11,12,13]$ and Hasumi $[8$, Corollary 7.2]. To do so, let $C_{0}(R)$ be the set of real continuous functions on $R$ which vanishes at infinity. When $k$ ranges over the class $C_{0}(R)$, the seminorms

$$
\|f\|_{k}=\sup \{|f(z) k(z)|: z \in R\}
$$


define a topology on $H^{\infty}(R)$, which is called the $\beta$ topology. This topology has been studied extensively by Rubel and Shields [17] and by Neville [13]. In particular, it is known that a subspace $M$ of $H^{\infty}(R)$ is $\beta$-closed if and only if $\{\hat{f}: f \in M\}$ is weak* closed in $H^{\infty}(d \chi)$. Now, we have the following.

Corollary 5.7. Let $M$ be a closed ( $\beta$-closed, if $p=\infty) H^{\infty}(R)$-submodule of $H^{p}(R)$. Then, $M=q H^{p}\left(R, \gamma^{-1}\right)$, where $q$ is the greatest common inner divisor of $M$ with character $\gamma$.

The following corollary is of interest from the point of view of the abstract Hardy class theory (cf. [5, Chapter V, Theorem 4.2]).

COROLlaRY 5.8. Let $1 \leqslant p \leqslant r \leqslant \infty$. Then $H^{p}(d \chi, \gamma) \cap L^{r}(d \chi)=H^{r}(d \chi, \gamma)$. If, in addition, $H^{r}(R, \gamma)$ is regular, then $H^{r}(R, \gamma)$ is dense in $H^{p}(d \chi, \gamma)$. In particular, $H^{r}(R)$ is dense in $H^{p}(R)$. (Note that $H^{r}(R)$ is regular because it contains constant functions.)

Finally, we obtain the following result due to Hasumi as a corollary of Theorem $5.1^{\prime}$.

Corollary 5.9 (HaSUmi [10]). Let $R$ be a hyperbolic Riemann surface of ParreauWidom type. Then $H^{\infty}(d \chi)$ is maximal among the weak* closed proper subalgebras of $L^{\infty}(d \chi)$.

Proof. We may assume that $R$ is regular. Let $\mathscr{Q}$ be a weak* closed subalgebra between $H^{\infty}(d \chi)$ and $L^{\infty}(d \chi)$. Since $H^{\infty}(d \chi) \subseteq \mathbb{Q}, \mathbb{Q}$ is an invariant subspace of $L^{\infty}(d \chi)$. By Theorem $5.1^{\prime}$, we must have $\mathcal{Q}=L^{\infty}(d \chi)$ if $\mathbb{Q}$ is doubly invariant. Suppose that $\mathbb{Q}$ is simply invariant. Then, $\mathscr{Q} \subseteq q H^{\infty}(d \chi, \gamma)$ for some $q \in L^{\infty}(d \chi)$, $|q|=1$ a.e., and $\gamma \in \pi(R)^{*}$. We may assume that the set $X=\left\{f \in H^{\infty}(R, \gamma)\right.$ : $q \hat{f} \in \mathbb{Q}\}$ is regular. Since $1 \in \mathbb{Q}, q \hat{q}^{\prime}=1$ a.e. on $\Delta$ for some $q^{\prime} \in X$. Hence, this $q^{\prime}$ is an inner function. Let $A=\left(1 / q^{\prime}\right) X$. Since $\mathcal{Q}=\{\hat{f}: f \in A\}$, and since $\mathcal{Q}$ is an algebra, it follows from Lemma 5.2 that $A$ is also an algebra. Hence, $A^{2} \subseteq A$, or $X^{2} \subseteq q^{\prime} X$. This means that $q^{\prime}$ is a common inner divisor of $X^{2}$. Since $X$ is regular, $q^{\prime}$ must be a constant inner function. Therefore, $H^{\infty}(R, \gamma)=H^{\infty}(R)$, and $\mathbb{Q}$ coincides with $H^{\infty}(d \chi)$.

6. Remarks on the identity (3.1) and examples. It is unknown whether the identity (3.1) holds for every regular hyperbolic Riemann surface of Parreau-Widom type. This identity, however, is necessary in order to obtain our invariant subspace theorem. More precisely, we have the following theorem.

THEOREM 6.1. Let $R$ be a regular hyperbolic Riemann surface of Parreau-Widom type. For $\gamma \in \pi(R)^{*}$ and $1 \leqslant p \leqslant \infty$, set

$$
m^{p}(\gamma)=\sup \left\{|f(\mathbf{O})|: f \in H^{p}(R, \gamma),\|f\|_{p} \leqslant 1\right\} .
$$

Then, the following conditions are mutually equivalent:

(a) Every $\beta$-closed ideal of $H^{\infty}(R)$ has the form $q H^{\infty}(R, \gamma)$, where $q$ is an inner function with the character $\gamma^{-1}$.

(b) The identity (3.1) holds. 
(c) $m^{p}(\gamma) m^{p^{\prime}}\left(\gamma^{-1} \tilde{\gamma}\right)=m^{1}(\tilde{\gamma})$, for every $\gamma \in \pi(R)^{*}$ and $1 \leqslant p, p^{\prime} \leqslant \infty, p^{-1}+p^{\prime-1}$ $=1$.

(d) For each $1 \leqslant p \leqslant \infty, m^{p}(\gamma)$ is continuous for $\gamma \in \pi(R)^{*}$.

(e) $\lim _{n} m^{\infty}\left(\gamma_{n}^{-1}\right)=1$, where $\gamma_{n}$ is the character of

$$
\exp \left(-\sum_{k>n}\left\{G\left(\cdot, a_{k}\right)+i^{*} G\left(\cdot, a_{k}\right)\right\}\right) \text {. }
$$

Assuming the theorem for a while, let us construct examples of Riemann surfaces satisfying the above conditions.

Let $R$ be an $m$-sheet branched full covering surface of the open unit disc $U$. Suppose that the projections of the branch points form the zero set of a Blaschke product on $U$. Let $\phi: R \rightarrow U$ be the projection. We may choose the point $\mathbf{O}$ so that $\phi(\mathbf{O})=0$. By Stanton [24], $R$ is of Parreau-Widom type and there are constants $0<r<1$ and $A>0$ such that

$$
\frac{1}{m} G_{0}(\phi(z), 0) \leqslant A G(z, \mathbf{O}) \text { for }|\phi(z)|>r .
$$

Here $G_{0}(w, 0)=-\log |w|$ is the Green function for $U$. From this inequality and the fact that $R$ is of Parreau-Widom type, it follows that the projections of the critical points $a_{k}$, counting their multiplicities, form the zero set of a Blaschke product on $U$. Let $B_{n}$ be the Blaschke product whose zero set is $\left\{\phi\left(a_{k}\right)\right\}_{k>n}$. For simplicity of notation, we define

$$
Z_{a}=\exp \left(-G(\cdot, a)+i^{*} G(\cdot, a)\right) .
$$

Since $B_{n} \circ \phi$ is a single-valued Blaschke product on $R, B_{n} \circ \phi / \Pi_{k>n} Z_{a_{k}}$ belongs to $H^{\infty}\left(R, \gamma_{n}^{-1}\right)$. Since $B_{n}(0) \rightarrow 1, m^{\infty}\left(\gamma_{n}^{-1}\right) \rightarrow 1$. Therefore, the surface $R$ satisfies the condition (e).

One can generalize the above reasoning and can prove the following:

(I) Let $R_{0}$ be a regular Riemann surface satisfying the conditions in the theorem and $R$ an $m$-sheet branched full covering surface of $R_{0}$. If the projections of the branch points form the zero set of a Blaschke product on $R_{0}$, then $R$ also satisfies the conditions.

In proving this, one may find that the Blaschke product $B_{n}$ may not be single valued on $R_{0}$. Multiplying a suitable element with the inverse character to $B_{n}$, one obtains a single valued function $B_{n}^{\prime}$. Use the condition (d) to prove $B_{n}^{\prime}(\phi(\mathbf{O})) \rightarrow 1$.

Following Voichick-Neville's examples (cf. [13, Chapter 8]), we show another way of constructing Riemann surfaces that satisfy the conditions in the theorem. Namely,

(II) Let $R_{0}$ be as in (I), and let $A_{k}(k \geqslant 1)$ be disjoint compact continua such that $R=R_{0} \backslash \cup_{k} A_{k}$ is connected. If there are points $b_{k}$ in $A_{k}$ with $\Sigma_{k} G_{0}\left(\mathbf{O}, b_{k}\right)<\infty$, then $R$ also satisfies the condition.

We briefly sketch the proof of this fact. Choose disjoint domains $R_{k}$ which contain $A_{k}$ and are surrounded by a finite number of closed curves. Let $\iota: R \rightarrow R_{0}$ be an inclusion mapping and $\iota_{*}: \mathcal{H}_{*}(R) \rightarrow \mathcal{H}_{*}\left(R_{0}\right)$ the induced homomorphism of homology groups with integer coefficients. Applying the Mayer-Vietoris exact sequences to the pair $\left(R_{0}, \cup_{k} R_{k}\right)$, we obtain an isomorphism

$$
\mathcal{H}_{1}(R) \cong \mathscr{F} \oplus \mathcal{G}
$$


where $\mathscr{F}=\operatorname{Im}\left(\iota_{*}\right)$ and $\mathcal{G}=\operatorname{Ker}\left(\iota_{*}\right)$. It is not difficult to see that $\mathcal{G}$ is the subgroup of $\mathcal{H}_{1}(R)$ generated by the closed 1-chains $\partial R_{k}, k \geqslant 1$. As in Neville [13, Lemma 8.2.1], there exist probability measures $\mu_{k}$ on $A_{k}$ such that $u_{k}=\int G_{0}(\cdot, b) d \mu_{k}(b)$ are bounded positive harmonic functions on $R$ and

$$
\begin{gathered}
\sum_{k} u_{k}(\mathbf{O})<\infty, \\
\int_{\partial R_{\jmath}}{ }^{*} d u_{k}= \begin{cases}-2 \pi & (j=k), \\
0 & (j \neq k) .\end{cases}
\end{gathered}
$$

Now let $\gamma \in \pi(R)^{*}$ and set $c_{k}=\arg \gamma\left(\partial R_{k}\right) / 2 \pi$, where $0 \leqslant c_{k}<1$. For $\varepsilon>0$, we fix $N=N(\varepsilon)$ with $\sum_{k>N} u_{k}(\mathbf{O})<\varepsilon$. Define

$$
u=\sum c_{k} u_{k}
$$

for $k$ satisfying either $k>N$ or $c_{k} \leqslant \frac{1}{2}$, and

$$
v=\sum\left(c_{k}-1\right) u_{k}
$$

for $k$ satisfying both $k \leqslant N$ and $c_{k}>\frac{1}{2}$. The first sum converges by (6.1), So, the function $u$ is positive harmonic on $R$. On the other hand, $v$ is a bounded harmonic function on $R$. Set $g=\exp \left(-u-v-i^{*} u-i^{*} v\right)$. By (6.2), the character $\gamma_{1}$ of $g$ agrees with $\gamma$ on $\mathcal{G}$. Set $\gamma_{2}=\gamma \gamma_{1}^{-1} \mid \mathcal{F}$. By the duality theorem of locally compact abelian groups, $\mathscr{F}^{*}$ is isomorphic to $\mathcal{H}_{1}\left(R_{0}\right)^{*} / \mathscr{F}^{\perp}$. Thus, we can extend $\gamma_{2}$ to a character $\gamma_{2}^{\prime} \in \mathcal{H}_{1}\left(R_{0}\right)^{*}=\pi\left(R_{0}\right)^{*}$. Since the quotient mapping from $\mathcal{H}_{1}\left(R_{0}\right)^{*}$ onto $\mathscr{F}^{*}$ is open, we can choose $\gamma_{2}^{\prime}$ so that $\gamma_{2}^{\prime}$ converges to 1 in $\pi_{1}\left(R_{0}\right)^{*}$ when $\gamma_{2}$ converges to 1 in $\mathscr{F}^{*}$. Let $f \in H^{\infty}\left(R_{0}, \gamma_{2}^{\prime}\right)$. Since $\partial R_{k}$ is chain homotopic to zero in $R, f$ has no periods along $\partial R_{k}$. Hence, $h=f g$ belongs to $H^{\infty}(R, \gamma)$. Since

$$
\|h\|_{\infty} \leqslant\|f\|_{\infty} \exp \left(\|v\|_{\infty}\right) \text { and }|h(\mathbf{O})|=|f(\mathbf{O}) g(\mathbf{O})|,
$$

it follows that

$$
m^{\infty}(R, \gamma) \geqslant m^{\infty}\left(R_{0}, \gamma_{2}^{\prime}\right) \exp (-u(\mathbf{O})-v(\mathbf{O})) \exp \left(-\|v\|_{\infty}\right) .
$$

Here $m^{\infty}(R, \gamma)$ and $m^{\infty}\left(R_{0}, \gamma\right)$ denote the quantity $m^{\infty}(\gamma)$ with respect to $R$ and $R_{0}$, respectively. If $\gamma \rightarrow 1$, then $c_{k}$ approaches either 0 or 1 for each $k$. So, $\lim \sup _{\gamma \rightarrow 1} u(\mathbf{O}) \leqslant \varepsilon$ and $\lim _{\gamma \rightarrow 1}\|v\|_{\infty}=0$. Moreover, since $\gamma_{1} \rightarrow 1, \gamma_{2} \rightarrow 1$, and so, $\gamma_{2}^{\prime} \rightarrow 1$, as noted above. By the condition (d), we have $m^{\infty}\left(R_{0}, \gamma_{2}^{\prime}\right) \rightarrow 1$. Hence, $\liminf _{\gamma \rightarrow 1} m^{\infty}(R, \gamma) \geqslant e^{-\varepsilon}$. Since $\varepsilon>0$ is arbitrary, $m^{\infty}(R, \gamma) \rightarrow 1$ as $\gamma \rightarrow 1$. Therefore, $R$ satisfies the condition (e).

Beginning with the open unit disc, one can construct a variety of Riemann surfaces satisfying the conditions in the theorem by repetitions of (I) and (II).

Now we turn to the proof of the theorem. In what follows, we assume that $R$ is a regular hyperbolic Riemann surface of Parreau-Widom type. It follows that

$$
m^{p}(\gamma) m^{p^{\prime}}\left(\gamma^{-1} \tilde{\gamma}\right) \geqslant \exp \left(-\sum_{k} G\left(\mathbf{O}, a_{k}\right)\right) \text { for } 1 \leqslant p, p^{\prime} \leqslant \infty, p^{-1}+p^{\prime-1}=1 .
$$


In fact, by Widom [23, Lemma and Theorem II], equality holds in (6.3) when $R$ is a finite bordered Riemann surface. The inequality (6.3) is obtained by a normal family argument with respect to the exhaustion $\{z \in R: G(z, \mathbf{O})>1 / n\}, n=1,2,3, \ldots$, of $R$. By [23, Theorem II], we also know that

$$
\inf _{\gamma} m^{p}(\gamma)=\exp \left(-\sum_{k} G\left(\mathbf{O}, a_{k}\right)\right), \quad 1 \leqslant p \leqslant \infty .
$$

LEMMA 6.2. If $H^{\infty}(R, \gamma)$ is regular, then $H^{\infty}\left(R, \gamma^{-1}\right)$ also is regular.

Proof. Let $q_{0}$ be the greatest common inner divisor of $H^{\infty}\left(R, \gamma^{-1}\right)$ and $\gamma_{0}$ the character of $q_{0}$. We have

$$
H^{\infty}\left(R, \gamma^{-1}\right)=q_{0} H^{\infty}\left(R, \gamma_{0}^{-1} \gamma^{-1}\right) .
$$

Now $H^{\infty}\left(\mathrm{R}, \gamma_{0}^{-1} \gamma^{-1}\right)$ is regular, and since

$$
H^{\infty}\left(R, \gamma_{0}\right) H^{\infty}\left(R, \gamma_{0}^{-1} \gamma^{-1}\right) \subseteq H^{\infty}\left(R, \gamma^{-1}\right),
$$

the inner factor of every element of $H^{\infty}\left(R, \gamma_{0}\right)$ must be divided by $q_{0}$. Thus, $H^{\infty}\left(R, \gamma_{0}\right)=q_{0} H^{\infty}(R)$. By induction, we prove $H^{\infty}\left(R, \gamma_{0}^{k}\right)=q_{0}^{k} H^{\infty}(R)$ for every positive integer. Suppose that this is true for $k$. Then,

$$
H^{\infty}\left(R, \gamma_{0}^{k+1}\right) H^{\infty}\left(R, \gamma_{0}^{-1} \gamma^{-1}\right) H^{\infty}(R, \gamma) \subseteq q_{0}^{k} H^{\infty}(R) .
$$

Since both $H^{\infty}\left(R, \gamma_{0}^{-1} \gamma^{-1}\right)$ and $H^{\infty}(R, \gamma)$ are regular, the inner factor of every element in $f$ in $H^{\infty}\left(R, \gamma_{0}^{k+1}\right)$ must be divided by $q_{0}^{k}$. That is, $f$ can be written in the form

$$
f=q_{0}^{k} g, \quad g \in H^{\infty}\left(R, \gamma_{0}\right) .
$$

Since $H^{\infty}\left(R, \gamma_{0}\right)=q_{0} H^{\infty}(R)$, we have $H^{\infty}\left(R, \gamma_{0}^{k+1}\right)=q^{k+1} H^{\infty}(R)$. Now, it follows that

$$
m^{\infty}\left(\gamma_{0}^{k}\right)=\left|q_{0}(\mathbf{O})\right|^{k}
$$

for every positive integer $k$. By (6.4), $q_{0}$ must be a constant inner function. Therefore, $H^{\infty}\left(R, \gamma^{-1}\right)$ is regular.

Now, we set

$$
\Lambda_{n}=\prod_{k>n} Z_{a_{k}} \text { and } \Lambda_{n}^{\prime}=Z_{\mathbf{o}} / \prod_{k=1}^{n} Z_{a_{k}}
$$

$\Lambda_{n}$ is the function in the condition (e). We denote by $\gamma_{n}^{\prime}$ the character of $\Lambda_{n}^{\prime}$. Note that $\Lambda_{n} \rightarrow 1$ and $\Lambda_{n}^{\prime} \rightarrow \Lambda_{*}$ as $n \rightarrow \infty$. We also set

$$
I_{n}(R)=\Lambda_{n} H^{\infty}\left(R, \gamma_{n}^{-1}\right) \text { and } K_{n}(R)=\Lambda_{n}^{\prime} H^{1}\left(R, \gamma_{n}^{\prime-1}\right)
$$

and denote by $I_{n}$ and $K_{n}$ the sets of boundary values of functions in $I_{n}(R)$ and $K_{n}(R)$, respectively.

Since we may choose any point of $R$ for $\mathbf{O}$, it follows from (6.4) that no $Z_{a}$ are a common inner divisor of any of $H^{\infty}(R, \gamma)$. Since $\Lambda_{n}$ belongs to $H^{\infty}\left(R, \gamma_{n}\right), H^{\infty}\left(R, \gamma_{n}\right)$ is regular, and hence, $H^{\infty}\left(R, \gamma_{n}^{-1}\right)$ is regular by the above lemma. This shows that (6.5) the greatest common inner divisor of $\bigcup_{n=1} I_{n}(R)$ is a constant inner function. 
LEMMA 6.3. Let $K_{0}$ be the $L^{1}(d \chi)$ closure of $\cup_{n=1} K_{n}$ and I the weak ${ }^{*}$ closure of $\cup_{n=1} I_{n}$ in $L^{\infty}(d \chi)$. Then,

$$
K_{0}=H^{\infty}(d \chi)^{\perp} \quad \text { and } \quad I=K^{1}(d \chi)^{\perp} .
$$

Proof. By [8, Theorem 4.2],

$$
K_{n} \subseteq H^{\infty}(d \chi)^{\perp}
$$

and it is obvious that

$$
I_{n}(R) K^{1}(R) \subseteq K_{n}^{1}(R) .
$$

Thus, if $f^{*} \in K_{0}^{\perp}$, then $\int \hat{g} \hat{h}^{*} d \chi=0$ for any $g \in K^{1}(R)$ and $h \in I_{n}(R)$. Hence, for every $h$ in $I_{n}(R), \hat{h} f^{*}$ is orthogonal to $K^{1}(d \chi)$. By the inverse Cauchy theorem, $\hat{h} f^{*}$ is the boundary values of a bounded analytic function, say $f$. So, $f^{*}=\hat{f} / \hat{h}$. By Lemma $5.2, f / h$ is a meromorphic function on $R$ which does not depend on the choice of $h$. By (6.5) and Lemma 5.3, $f / h$ is a bounded analytic function. This proves $K_{0}^{\perp} \subseteq$ $H^{\infty}(d \chi)$. The reverse inclusion follows from (6.6).

For the second identity, we have $K^{1}(d \chi) \subseteq I^{\perp}$ by (6.6) and (6.7). If $g^{*} \in I^{\perp}$, the $\int g^{*} \hat{h} d \chi=0$ for every $h \in I_{n}(R)$. Since $I_{n}(R)$ is an ideal of $H^{\infty}(R), g^{*} \hat{h}$ belongs to $H^{\infty}(d \chi)^{\perp}=K_{0}$. Since $K_{0} \subseteq K^{1}(d \chi), g^{*} \hat{h}=\hat{g} / \hat{\Lambda}$ for some $g \in H^{1}\left(R, \gamma_{*}^{-1}\right)$. So, $\hat{\Lambda}_{*} g^{*}=\hat{g} / \hat{h}$. Again, by (6.5) and Lemma 5.3, we deduce that $g / h$ is in $H^{1}\left(R, \gamma_{*}\right)$. Therefore, $g^{*} \in K^{1}(d \chi)$, and hence $I^{\perp} \subseteq K^{1}(d \chi)$. This proves the lemma.

Proof of Theorem 6.1. We first show that the identity $I=H^{\infty}(d \chi)$ implies the identity (3.1). If $I=H^{\infty}(d \chi)$, we have $H^{\infty}(d \chi)^{\perp}=K^{1}(d \chi)$ by the preceding lemma. Let $f \in H^{1}(R, \tilde{\gamma})$. Set $g=f / \tilde{\Lambda}$. Since $g-g(\mathbf{O}) \in K^{1}(R), \int(\hat{g}-g(\mathbf{O})) \cdot 1 d \chi$ $=0$. Hence,

$$
\int \hat{g} d \chi=g(\mathbf{O})=f(\mathbf{O}) / \tilde{\Lambda}(\mathbf{O})
$$

This implies

$$
|f(\mathbf{O})| \leqslant|\tilde{\Lambda}(\mathbf{O})|\|\hat{g}\|_{1}=|\tilde{\Lambda}(\mathbf{O})|\|f\|_{1} .
$$

It follcws that $m^{1}(\tilde{\gamma}) \leqslant|\tilde{\Lambda}(\mathbf{O})|$, and we obtain the identity (3.1) by (6.4).

Now, if (a) holds, then $I=H^{\infty}(d \chi)$ by (6.5). Hence, (b) holds. Conversely, if (b) holds, then (a) follows from Corollary 5.7. Therefore, (a) and (b) are equivalent.

If $f \in H^{p}(R, \gamma)$ and $g \in H^{p^{\prime}}\left(R, \gamma^{-1} \tilde{\gamma}\right)$, then $f g \in H^{1}(R, \tilde{\gamma})$ and $\|f g\|_{1} \leqslant$ $\|f\|_{p}\|g\|_{p^{\prime}}$. Thus, we have

$$
m^{p}(\gamma) m^{p^{\prime}}\left(\gamma^{-1} \tilde{\gamma}\right) \leqslant m^{1}(\tilde{\gamma})
$$

Therefore, (c) follows from (b) by (6.3) and (6.4).

Now suppose that (c) holds. Since $m^{\infty}(\gamma) \leqslant 1, m^{1}\left(\gamma^{-1} \tilde{\gamma}\right) \geqslant m^{1}(\tilde{\gamma})$. On the other hand, $\lim \sup _{\gamma \rightarrow 1} m^{1}\left(\gamma^{-1} \tilde{\gamma}\right) \leqslant m^{1}(\tilde{\gamma})$ follows from a normal family argument. Hence, $\lim _{\gamma \rightarrow 1} m^{1}\left(\gamma^{-1} \tilde{\gamma}\right)=m^{1}(\tilde{\gamma})$, and it follows from the formula in (c) that $\lim _{\gamma \rightarrow 1} m^{\infty}(\gamma)$ $=1$. Now let $\gamma_{0} \in \pi(R)^{*}$. Since $H^{p}\left(R, \gamma_{0}\right) H^{\infty}\left(R, \gamma_{0}^{-1} \gamma\right) \subseteq H^{p}(R, \gamma)$, we have

$$
m^{p}\left(\gamma_{0}\right) m^{\infty}\left(\gamma_{0}^{-1} \gamma\right) \leqslant m^{p}(\gamma) \text {. }
$$

Thus, $\liminf _{\gamma \rightarrow \gamma_{0}} m^{p}(\gamma) \geqslant m^{p}\left(\gamma_{0}\right)$, because $\gamma_{0}^{-1} \gamma \rightarrow 1$. Also, by a normal family argument, we have $\lim \sup _{\gamma \rightarrow \gamma_{0}} m^{p}(\gamma) \leqslant m^{p}\left(\gamma_{0}\right)$. Hence, $m^{p}(\gamma)$ is continuous for $\gamma \in \pi(R)^{*}$. 
It is trivial that (d) implies (e). If $m^{\infty}\left(\gamma_{n}^{-1}\right) \rightarrow 1$ as $n \rightarrow \infty$, take a function $f_{n}$ in $H^{\infty}\left(R, \gamma_{n}^{-1}\right)$ with $\left|f_{n}\right| \leqslant 1$ and $\left|f_{n}(0)\right|=m^{\infty}\left(\gamma_{n}^{-1}\right)$. Then, $\Lambda_{n} f_{n}$ converges to 1 pointwise boundedly on $R$. Thus, $1 \in I$, so, $I=H^{\infty}(d \chi)$. This shows that (e) implies (b) by the first part of the proof. This completes the proof.

REMARK. There is another way of proving our direct Cauchy theorem. As we have seen, the identity (3.1) implies the condition (e). Using the condition (e), one can prove the direct Cauchy theorem in a similar way to that of Hasumi [8].

ADDENDUM. I recently found an example of a plane domain that is of ParreauWidom type but does not have condition (3.1).

\section{REFERENCES}

1. A. Beurling, On two problems concerning linear transformations in Hilbert space, Acta Math. 81 (1949), 239-255.

2. M. Brelot and G. Choquet, Espaces et Lignes de Green, Ann. Inst. Fourier (Grenoble) 3 (1951), 199-263

3. C. Constantinescu and A. Cornea, Ideal Ränder Riemannscher Flächen, Springer-Verlag, BerlinGöttingen-Heidelberg, 1963.

4. P. L. Duren, Theory of $H^{p}$ spaces. Academic Press, New York-London, 1970.

5. T. W. Gamelin, Uniform algebras, Prentice-Hall, Englewood Cliffs, N. J., 1969.

6. F. Forelli, Bounded holomorphic functions and projections, Illinois J. Math. 10 (1966), 367-380.

7. M. Hasumi, Invariant subspace theorems for finite Riemann surfaces, Canad. J. Math. 18 (1966), $240-255$

8. __ Invariant subspaces on open Riemann surfaces, Ann. Inst. Fourier (Grenoble) 24-4 (1974), 241-286.

9. __ Invariant subspaces on open Riemann surfaces. II, Ann. Inst. Fourier (Grenoble) 26-2 (1976), 273-299.

10. Weak-star maximality of $H^{\infty}$ for surfaces of Parreau-Widom type, preprint.

11. C. W. Neville, Ideals and submodules of analytic functions on infinitely connected plane domains, Thesis, University of Illinois at Urbana-Champaign, Urbana, Ill., 1972.

12. Invariant subspaces of Hardy classes on infinitely connected plane domains, Bull. Amer. Math. Soc. 78 (1972), 857-860.

13. Invariant subspaces of Hardy classes on infinitely connected open surfaces, Mem. Amer. Math. Soc., No. 160.

14. M. Parreau, Théorème de Fatou et problème de Dirichlet pour les lignes de Green de certaines surfaces de Riemann, Ann. Acad. Sci. Fenn. Ser. A I, no. 250/255 (1958), 1-8.

15. A. H. Read, $A$ converse of Cauchy's theorem and application to extremal problems, Acta Math. 100 (1958), 1-22.

16. H. L. Royden, The boundary values of analytic and harmonic functions, Math. Z. 78 (1962), 1-24.

17. L. A. Rubel and A. L. Shields, The space of bounded analytic functions on a region. Ann. Inst. Fourier (Grenoble) 16 (1966), 235-277.

18. D. Sarason, The $I^{\prime \prime}$ spaces of an annulus, Mem. Amer. Math. Soc., No. 56, 1965.

19. T. P. Srinivasan, Doubly invariant subspaces, Pacific J. Math. 14 (1964), 701-707.

20. Simply invariant subspaces and generalized analytic functions, Proc. Amer. Math. Soc. 16 (1965), 813-818.

21. M. Voichick, Ideals and invariant subspaces of analytic functions, Trans. Amer. Math. Soc. 111 (1964), 493-512.

22. __ Invariant subspaces on Riemann surfaces, Canad. J. Math. 18 (1966), 399-403.

23. H. Widom, $\mathcal{H}_{p}$ sections of vector bundles over Riemann surfaces, Ann. of Math. (2) 94 (1971), 304-324.

24. C. M. Stanton, Bounded analytic functions on a class of open Riemann surfaces, Pacific J. Math. 59 (1975), 557-565.

Department of Mathematics, University of California, Los Angeles, California 90024

DePartment of Mathematics, Ibaraki University, Mito, Ibaraki, JaPAN

Current address: Department of Mathematics, Hokkaido University, Sapporo, Japan 